\title{
Correction to: Assessment of the $1 \%$ of Patients with Consistent $<15 \%$ Reduction in Low-Density Lipoprotein Cholesterol: Pooled Analysis of 10 Phase 3 ODYSSEY Alirocumab Trials
}

\author{
Harold E. Bays ${ }^{1} \cdot$ Robert S. Rosenson ${ }^{2} \cdot$ Marie T. Baccara-Dinet ${ }^{3} \cdot$ Michael J. Louie $^{4} \cdot$ Desmond Thompson $^{4}$. \\ G. Kees Hoving ${ }^{5}$
}

Published online: 27 April 2018

(C) Springer Science+Business Media, LLC, part of Springer Nature 2018

\section{Correction to: Cardiovasc Drugs Ther https://doi.org/10.1007/s10557-018-6784-z}

The original version of this article unfortunately contained a mistake in the Discussion section. The line that currently reads "During this 4-week period while off PCSK9 monoclonal antibody therapy, the medical staff should ensure an accurate 4-week pill count of concurrently administered statin and/ or other lipid-altering drugs." should be changed to "During this 4-week stabilization period (while off PCSK9 monoclonal antibody therapy for at least 8 weeks), the medical staff should ensure an accurate 4-week pill count of concurrently administered statin and/or other lipid-altering drugs."

The online version of the original article can be found at https://doi.org/ 10.1007/s10557-018-6784-z

Harold E. Bays

hbaysmd@outlook.com

1 Departments of Epidemiology \& Medicine, Louisville Metabolic and Atherosclerosis Research Center (L-MARC), 3288 Illinois Avenue, Louisville, KY 40213, USA

2 Cardiometabolics Unit, Mount Sinai Heart, Icahn School of Medicine at Mount Sinai, New York, NY, USA

3 Clinical Development, R\&D, Sanofi, Montpellier, France

4 Regeneron Pharmaceuticals, Inc., Tarrytown, NY, USA

5 Department of Vascular Medicine - Internal Medicine Academic Medical Center, University of Amsterdam,

Amsterdam, The Netherlands 